\title{
Polymerization of DL-Phenylalanine $N$-Carboxyanhydride by Multifunctional Pyridine Derivatives
}

\author{
Yukio Imanishi, Shoji Nagaoka, and Toshinobu Higashimura \\ Department of Polymer Chemistry, Kyoto University, Yoshida, \\ Sakyo-ku, Kyoto 606, Japan.
}

(Received November 15, 1972)

\begin{abstract}
Polymerizations of DL-phenylalanine $N$-carboxyanhydride (NCA) by pyridine, 2,2'-bipyridine, 4,4'-bipyridine, 2,2' $2^{\prime \prime}$-tripyridine, $2,2^{\prime}, 2^{\prime \prime}, 2^{\prime \prime \prime}$-tetrapyridine were carried out in nitrobenzene at $35^{\circ} \mathrm{C}$. When the aprotic base was in excess of NCA, the initial polymerization rate increased with increasing the basicity of the aprotic base. When NCA was in excess of the aprotic base, 2,2'-bipyridine led to the fastest polymerization. Since pyridine groups act as adsorption sites as well as catalytic sites, a cooperative function of two pyridine groups in bifunctional $2,2^{\prime}$-bipyridine is suggested. LCAO-MO calculations showed the possibility that two reacting species are adsorbed on $2,2^{\prime}$-bipyridine. Since they are arranged suitably for intramolecular collisions, the reaction rate increases. In the polymerization of DL-phenylalanine NCA by $n$-hexylamine in the presence of $2,2^{\prime}$-bipyridine as a matrix, the same sort of acceleration was also observed.
\end{abstract}

KEY WORDS DL-Phenylalanine NCA / Pyridine / 2,2'-Bipyridine / Cooperative Function / LCAO-MO Calculation /

The polymerization of DL-phenylalanine $N$ carboxyanhydride (NCA) by poly(2-vinylpyridine) has been studied in our laboratory., The NCA is adsorbed on the polymer chain by hydrogen bonding, activated by the pyridyl group, and reacts with other NCA adsorbed on the same polymer chain. Thus, the polymerization by the polymer catalyst is much faster than that by a monometric catalyst (pyridine or $\alpha$-picoline) having a similar base strength. Furthermore, the effect of the degree of polymerization (DP) of the polymer catalyst on the polymerization rate was studied in the detail, ${ }^{3}$ and it was found that pyridine groups along the polymer chain cooperate in hydrogen bonding with NCA and in the intramolecular collisions between adsorbed species. It occurred to us that the cooperation of pyridine groups in proximity should be investigated in more detail with a simple catalyst system. For this purpose, pyridine, bipyridines, tripyridine, and tetrapyridine were used as catalysts for the polymerization of DL-phenylalanine NCA. These catalysts have adsorption sites as well as catalytic sites, and are suited for a precise investigation of the cooperation among functional groups without the difficulties associated with the use of a polymer catalyst, such as viscosity, heat dissipation, etc.

\section{EXPERIMENTAL}

\section{Reagents}

DL-Phenylalanine NCA was synthesized by phosgenation of DL-phenylalanine. ${ }^{3}$ Aprotic base catalysts used were pyridine, 2,2'-bipyridine, $4,4^{\prime}$; bipyridine, $2,2^{\prime}, 2^{\prime \prime}$-tripyridine, $2,2^{\prime}, 2^{\prime \prime}, 2^{\prime \prime \prime}$ tetrapyridine, and poly(2-vinylpyridine). Pyridine was distilled from $\mathrm{KOH}$ and stored in a sealed ampoule, bp $115-116^{\circ} \mathrm{C} . \quad 2,2^{\prime}$-Bipyridine was recrystallized from petroleum ether, $\mathrm{mp} 70$ $71^{\circ} \mathrm{C}$. Other pyridines were commercial guaranteed reagent and used without further purification. Atactic poly(2-vinylpyridine) was obtained by radical polymerization. ${ }^{3}$

The normal-amine-type catalyst used was $n$ hexylamine, which was distilled over $\mathrm{CaH}_{2}$ and stored in a sealed ampoule, bp $128-129^{\circ} \mathrm{C}$.

Purifications of the solvents for polymerization (nitrobenzene) and for NCA purification (ethyl 
acetate and petroleum ether) have been previously reported. ${ }^{4}$

\section{Procedure}

All polymerizations were carried out in nitrobenzene solution at $35^{\circ} \mathrm{C}$ under a nitrogen stream. The polymerization rate was determined from the amount of carbon dioxide evolved in the polymerization. The apparatus and the method of determination have been reported by Patchornik previously. ${ }^{5}$

The equilibrium constant for hydrogen-bond formation between catalyst and NCA in methylene chloride was determined by infrared spectroscopy. ${ }^{6}$

Total $\pi$-electron density on the nitrogen atom of the aprotic bases was calculated by the simple Hückel method.

\section{RESULTS AND DISCUSSION}

\section{Polymerization of DL-Phenylalanine NCA by} Multifunctional Pyridine Derivatives

The polymerization of DL-phenylalanine NCA by pyridine derivatives is thought to proceed according to the "activated-NCA mechanism" which was first proposed by Bamford. ${ }^{7,8}$ In this mechanism the basicity of initiating amine is most important, and more basic amines usually lead to faster polymerizations. ${ }^{9}$ In the present polymerizations several characteristics of the activated-NCA mechanism ${ }^{8}$ were observed. However, more basic amines did not always give a faster polymerization. Figure 1 shows the

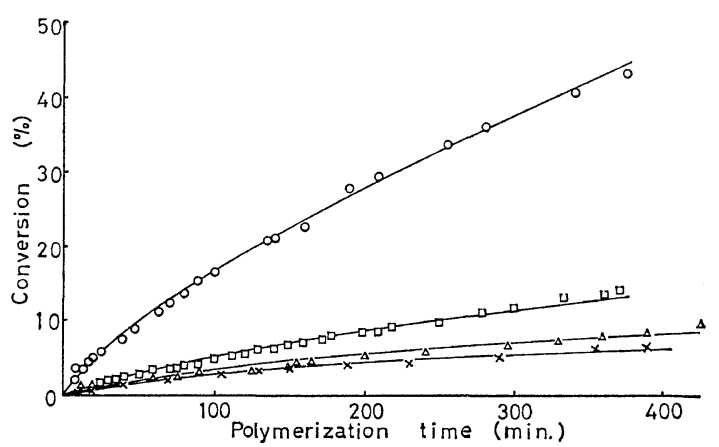

Figure 1. Polymerization of DL-phenylalanine NCA $(M)$ by pyridine derivatives $(I)$ at $M / I=0.2$ : nitrobenzene solution; $35^{\circ} \mathrm{C}$; $[\mathrm{NCA}]_{0}, 0.2 \mathrm{~mol} / l ; \bigcirc$, pyridine; $\triangle$, 2,2'-bipyridine; $\square$, 4,4-bipyridine; $\times$, $2,2^{\prime}, 2^{\prime \prime}$-tripyridine ( $\left.[\mathrm{NCA}]_{0}, 0.1 \mathrm{~mol} / l\right)$.

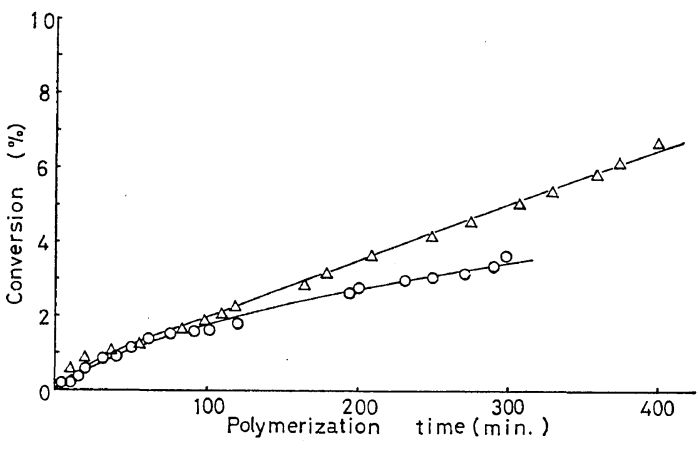

Figure 2. Polymerization of DL-phenylalanine NCA $(M)$ by pyridine derivatives $(I)$ at $M / I=5$ : conditions and notations the same as in Figure 1.

time-conversion curves for the polymerization under the condition that excess amines over NCAs are present. Throughout this paper the amine concentration refers to the concentration of pyridine residues. Under this condition more basic amines ( $c f$. Table I) give a faster polymerization. On the other hand, Figure 2 shows the time-conversion curves for the polymerization under the condition that excess NCAs over amines are present. Under this condition, less basic $2,2^{\prime}$-bipyridine gives a faster polymerization than more basic pyridine.

Under a variety of NCA-to-amine ratios the initial polymerization rate $\left(R_{\mathrm{p}}\right)$ was determined. $R_{\mathrm{p}}$ was determined from the slope of the firstorder plot of monomer consumption when it became a straight line (stationary state) after a short period at the very initial stages. In Figure $3, R_{\mathrm{p}}$ is plotted against the logarithm of the NCA-to-amine ratio $(M / I)$ for various pyridine derivatives whose basicities $\left(\mathrm{p} K_{\mathrm{a}}\right.$ value) are compared in Table I. 2,2 $2^{\prime}, 2^{\prime \prime}, 2^{\prime \prime \prime}$-Tetrapyridine initiated a very slow polymerization of DLphenylalanine NCA, so the experimental data are not shown in Figure 3. At low $M / I$ ratios, pyridine leads to a faster polymerization than 2,2'-bipyridine. Keeping $M$ constant $(0.2 \mathrm{~mol} / \mathrm{l})$ and decreasing $I, R_{\mathrm{p}}$ for pyridine decreases sharply but $R_{\mathrm{p}}$ for $2,2^{\prime}$-bipyridine does not change significantly. As a consequence, at large $M / I$ ratios, 2,2'-bipyridine initiates a faster polymerization than pyridine. Other pyridine derivatives, such as $4,4^{\prime}$-bipyridine, $2,2^{\prime}, 2^{\prime \prime}$-tripyridine, and $2,2^{\prime}, 2^{\prime \prime}, 2^{\prime \prime \prime}$-tetrapyridine behaved as expected 


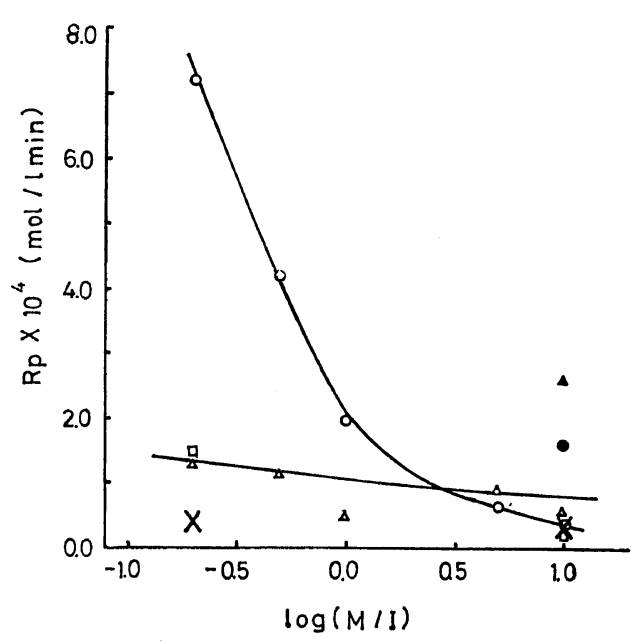

Figure 3. Initial rate of polymerization $\left(R_{\mathrm{p}}\right)$ of DL-phenylalanine NCA $(M)$ by pyridine derivatives (I) for various $M / I$ ratios: conditions and notations the same as in Figure 1; $\Delta,[\mathrm{NCA}]_{0}, 0.4 \mathrm{~mol} / l$, [2,2'-bipyridine], $0.04 \mathrm{~mol} / l ; 0,[\mathrm{NCA}]_{0}, 0.4 \mathrm{~mol} / l$, [pyridine], $0.04 \mathrm{~mol} / l$.

from their basicity. They never exceeded pyridine at any $M / I$ ratios studied.

The situation becomes clearer in the polymerization where both monomer and initiator concentrations are doubled while keeping $M / I$ constant $(M=0.4 \mathrm{~mol} / l, I=0.04 \mathrm{~mol} / l)$. In this case much faster polymerization takes place

and $\Delta$ in Figure 3 ), and the peculiarity of $2,2^{\prime}-$ bipyridine is clearly indicated.

It is suggested in Figure 3 that at large $M / I$ ratios some cooperation of two pyridine groups is operative with $2,2^{\prime}$-bipyridine, which compensates its low basicity and gives an abnormally high polymerization rate. The mechanism of cooperation is considered below.

Equilibrium Constant for Hydrogen-Bond Formation between DL-Phenylalanine NCA and Pyridine Derivatives

In multifunctional pyridine derivatives, pyridine groups act as adsorption sites as well as catalytic sites. ${ }^{1-3}$ The equilibrium constant $(K)$ for the hydrogen-bond formation should be determined, because it may have some bearing on the cooperative function of the catalyst. Under the various concentrations of NCA $(M=0.01-$ $0.03 \mathrm{~mol} / l)$ and pyridines $(I=0.01-0.04 \mathrm{~mol} / l)$, the intensity of $\mathrm{N}-\mathrm{H}$ stretching absorption at
Table I. Equilibrium constant $K$ for hydrogenbond formation between DL-phenylalanine NCA and various pyridine derivatives

\begin{tabular}{|c|c|c|}
\hline Pyridine derivatives & $\mathrm{p} K_{\mathrm{a}}$ & $K^{\mathrm{a}}, l / \mathrm{mol}$ \\
\hline Pyridine & $5.17^{10}$ & 15.0 \\
\hline $2,2^{\prime}$-Bipyridine & $4.44^{11}$ & 1.07 \\
\hline 4,4'-Bipyridine & $4.82^{11}$ & 3.44 \\
\hline $2,2^{\prime}, 2^{\prime \prime}-$ Tripyridine ${ }^{b}$ & $7.10,{ }^{12} \quad 4.35^{13}$ & 1.20 \\
\hline Poly(2-vinylpyridine) & $3.10^{14}$ & 4.52 \\
\hline
\end{tabular}

a In $\mathrm{CH}_{2} \mathrm{Cl}_{2}$ at room temperature.

b In ref $12 \mathrm{p} K_{\mathrm{a}}=7.10$ is reported; in ref $13 \mathrm{p} K_{\mathrm{a}}=$ 4.35 is reported although ref 12 is referred to; for the discussion in this paper the latter value was adopted as more reasonable.

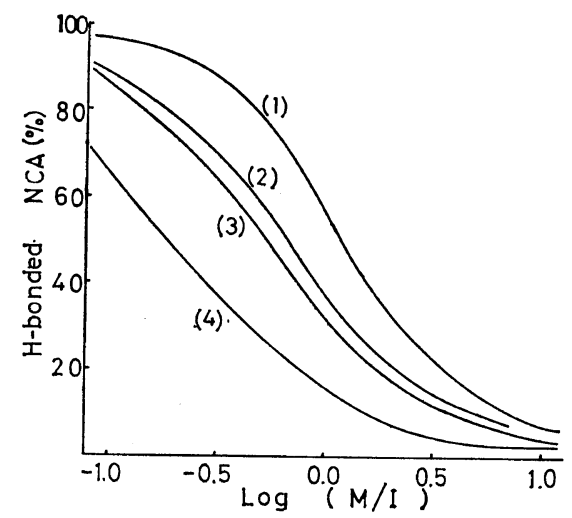

(a)

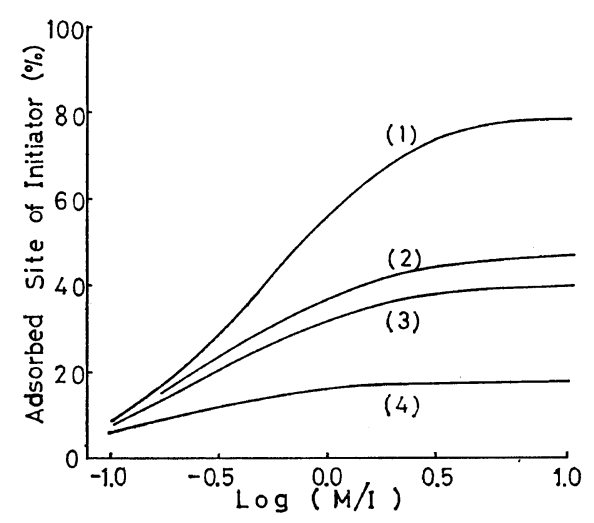

(b)

Figure 4. Percentages of (a) hydrogen-bonded NCAs and (b) hydrogen-bonded pyridine groups for various concentration ratios: 1, pyridine; 2 , poly(2-vinylpyridine); 3, 4,4'-bipyridine; 4, 2,2'bipyridine. 
$3437 \mathrm{~cm}^{-1}$, which is free from hydrogen bonding, was measured. For each run $K$ was determined. $K$ values were averaged, the mean deviation was calculated, and the mean $K$ value was determined. $K$ values for various pyridine derivatives are shown in Table I, together with their $\mathrm{p} K_{\mathrm{a}}$ values. Data for poly(2-vinylpyridine) are also shown for comparison. It is seen in Table I that the more basic amines form more hydrogen bonds with the NCA, except for the polymer. Therefore the peculiarity of $2,2^{\prime}$-bipyridine is not simply related to the formation of hydrogen bonds.
Using $K$ values listed in Table $\mathrm{I}$, the fractions of hydrogen-bonded NCA and of hydrogenbonded pyridine groups can be calculated for various conditions. Those percentages are shown in Figure 4. At large $M / I$ ratios with $2,2^{\prime}$ bipyridine the bulk of the NCAs is free from hydrogen bonding and the major fraction of adsorption sites (pyridine groups) is also free from hydrogen bonding. If free adsorption sites are in some way activated for hydrogen bonding, it might be possible that two adsorption sites in $2,2^{\prime}$-bipyridine are equally occupied during the reaction (Scheme 1).

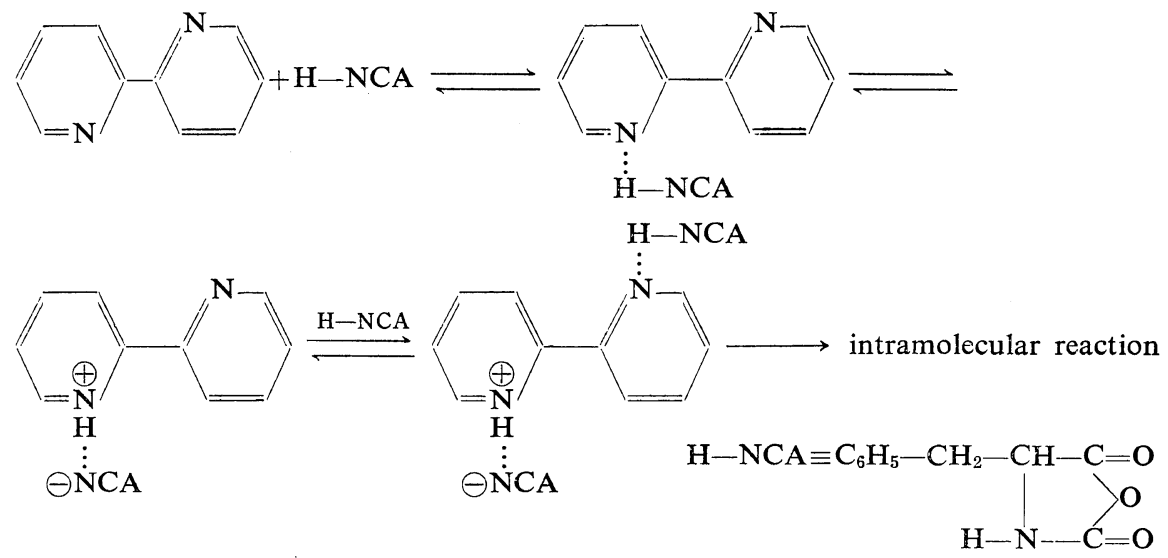

Scheme 1.

In order for $2,2^{\prime}$-bipyridine to show this sort of cooperation, the protonation should not inhibit the subsequent NCA adsorption. To check this the hydrogen-bond formation between $2,2^{\prime}$-bipyridine $(0.2-0.3 \mathrm{~mol} / l)$ and 5-ethyl-3methyl-5-phenyl hydantoin $(0.014-0.029 \mathrm{~mol} / \mathrm{l})$ (a model compound for DL-phenylalanine NCA, which is unstable against acetic acid) was investigated either in the absence (equilibrium constant $K_{1}$ ) or in the presence (equilibrium constant $K_{2}$ ) of acetic acid (half the concentration of $2,2^{\prime}$-bipyridine). In the presence of acetic acid one of the nitrogen pair of $2,2^{\prime}$-bipyridine will be protonated and then the hydantoin is adsorbed on to the other member of the nitrogen pair. The equilibrium constants at room temperature in methylene chloride are

$K_{1}=0.44 l / \mathrm{mol}$ without acetic acid

$K_{2}=0.31 \mathrm{l} / \mathrm{mol}$ with acetic acid
The protonation does not seem to affect the subsequent hydrogen bonding of 2,2'-bipyridine significantly.

The experimental findings described in this section suggest that there is some possibility for two NCA molecules to be adsorbed on two nitrogen atoms of $2,2^{\prime}$-bipyridine.

\section{Structure and Electronic State of Multifunctional Pyridine Derivatives}

It is known that the two pyridine groups of 2,2 -bipyridine assume a planar trans conformation in solution, and that a pyridine ring twists around the bond connecting $\mathrm{C}_{6}$ and $\mathrm{C}_{7}$ (see Scheme 2) by $80-100^{\circ}$ on monoprotonation and by $30^{\circ}$ on hydrogen bonding. ${ }^{15}$ For the cooperation depicted in Scheme 1 to operate, the basicity of the second pyridine group is very important and should be affected by the rotation around the $\mathrm{C}_{6}-\mathrm{C}_{7}$ bond. The total $\pi$-electron 


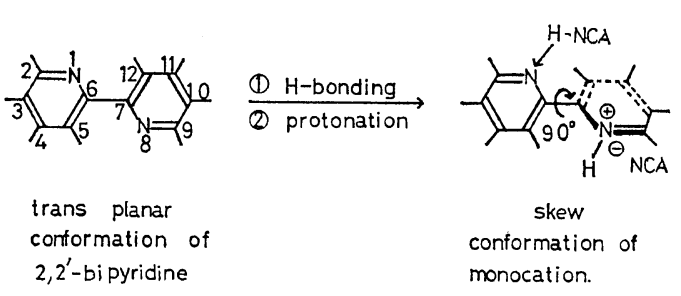

Scheme 2.

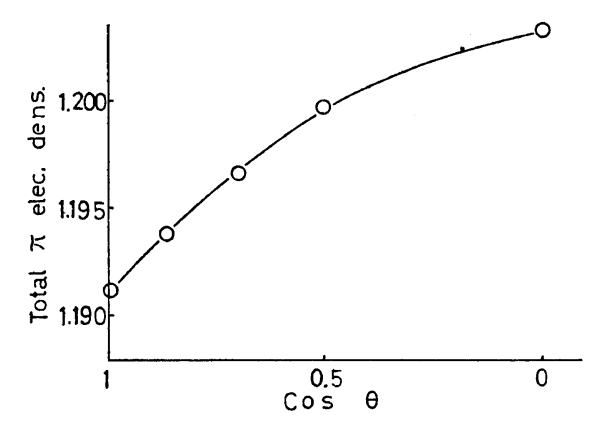

Figure 5. Total $\pi$-electron densities on $\mathrm{N}_{1}$ and the rotation angle around $\mathrm{C}_{6}-\mathrm{C}_{7}$ bond: parameters used for calculation ${ }^{16,17}$

X Coulomb Coulomb integral Resonance inte$\mathrm{X}$ integral for adjacent $\mathrm{C}$ gral for $\mathrm{C}-\mathrm{X}$

$$
\begin{array}{llll}
\searrow \mathrm{N} & \alpha+0.6 \beta & \alpha+0.1 \beta & \beta \\
\bigwedge^{\oplus} \alpha+2.0 \beta & \alpha+0.1 \beta & \beta
\end{array}
$$

densities of $\mathrm{N}_{1}$ were calculated according to LCAO-MO method with differently twisted monoprotonated 2,2'-bipyridines. Parameters used are given in Figure 5 together with the results of the calculation. In the calculation by a simple Hückel method, cis and trans conformations of the two pyridine groups were not discriminated.

When $N_{8}$ is hydrogen-bonded and further protonated, the total electron density of $\mathrm{N}_{1}$ decreases from 1.2090 to 1.1912 , but as a consequence of the twist around the $\mathrm{C}_{6}-\mathrm{C}_{7}$ bond it increases from 1.1912 to 1.2032 , which is equal to that of pyridine, that is, $\mathrm{N}_{1}$ is activated for hydrogen-bond formation. Hence, two reactive species are conceivably adsorbed on the same $2,2^{\prime}$-bipyridine.

It is quite interesting that this type of adsorption to $2,2^{\prime}$-bipyridine leads to rate acceleration whereas that to $4,4^{\prime}$-bipyridine does not. Presumably, the two reactive species adsorbed on
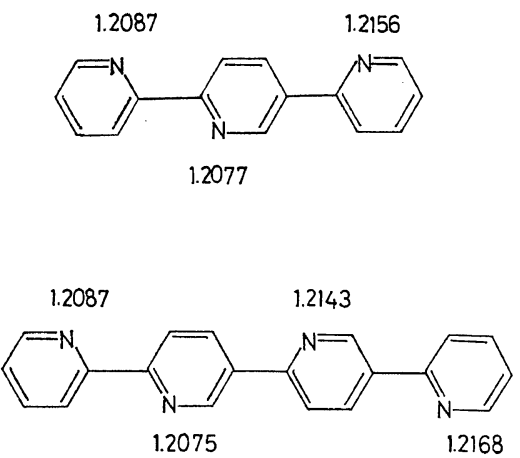

Figure 6. Total $\pi$-electron densities on nitrogen atoms of $2,2^{\prime}, 2^{\prime \prime}$-tripyridine and $2,2^{\prime}, 2^{\prime \prime}, 2^{\prime \prime \prime}$-tetrapyridine.

a skewed $2,2^{\prime}$-bipyridine are favorably arranged for intramolecular collisions. Collisions between species adsorbed on the two pyridine groups of 4,4'-bipyridine would be sterically difficult.

$2,2^{\prime}, 2^{\prime \prime}$-Tripyridine and $2,2^{\prime}, 2^{\prime \prime}, 2^{\prime \prime \prime}$-tetrapyridine possess two pyridine groups at the sterically favored arrangement (see Figure 6: N, 1.2087; $\mathrm{N}, 1.2077$ of $2,2^{\prime}, 2^{\prime \prime}$-tripyridine; N, 1.2087; N, 1.2075 of $2,2^{\prime}, 2^{\prime \prime}, 2^{\prime \prime \prime}$-tetrapyridine). Unfortunately, these groups are not electronically favored for adsorption of NCA and adsorption occurs on other nitrogens (see Figure 6: N, 1.2156 of $2,2^{\prime}, 2^{\prime \prime}$-tripyridine; $N, 1.2168$ of $2,2^{\prime}, 2^{\prime \prime}, 2^{\prime \prime \prime}$ tetrapyridine), so that no cooperative function was observed.

\section{Cooperative Function in Propagation Reaction}

The above consideration shows the possibility that two NCA molecules might be adsorbed to $2,2^{\prime}$-bipyridine and be apt to collide intramolecularly. If this is true an acceleration should be observed in the polymerization of DL-phenylalanine NCA by $n$-hexylamine in the presence of $2,2^{\prime}$ bipyridine. In the presence of $n$-hexylamine

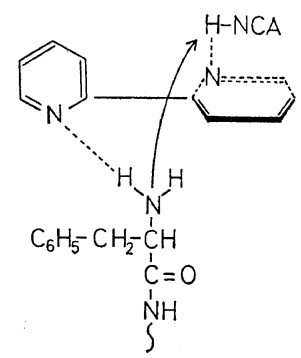

Scheme 3.

Polymer J., Vol. 4, No. 6, 1973 


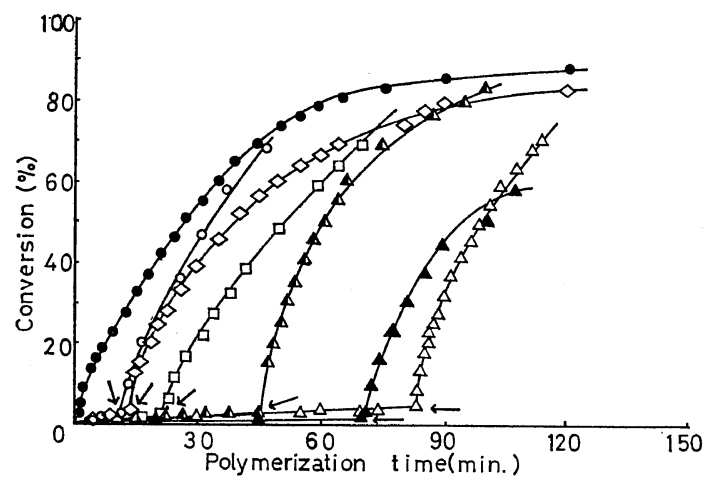

Figure 7. Polymerization of DL-phenylalanine NCA by $n$-hexylamine in the presence of pyridine derivatives: nitrobenzene solution, $35^{\circ} \mathrm{C},[\mathrm{NCA}]_{0}$, $0.2 \mathrm{~mol} / l$, [ $n$-hexylamine], $0.02 \mathrm{~mol} / l ; \bullet$, no pyridine derivatives; $\bigcirc$, [pyridine], $1.0 \mathrm{~mol} / l ; \Delta, \Delta$, [2,2'-bipyridine $], \quad 1,0 \mathrm{~mol} / l ; \quad \Delta, \quad\left[2,2^{\prime}\right.$-bipyridine $]$, $0.02 \mathrm{~mol} / l ; \square,\left[4,4^{\prime}\right.$-bipyridine], $1.0 \mathrm{~mol} / l ; \diamond$, [poly(2-vinylpyridine)], $1.0 \mathrm{~mol} / l$; the addition of $n$ hexylamine is indicated by an arrow.

$(0.02 \mathrm{~mol} / \mathrm{l})$ and a weak tertiary amine such as pyridine derivative $(1.0 \mathrm{~mol} / l)$, the polymerization is almost completely initiated by $n$-hexylamine, and the normal-amine-type propagation is concerned. ${ }^{8} \quad 2,2^{\prime}$-Bipyridine serves only as a matrix, and the contribution from the propagation reaction shown in Scheme 3 is expected.

Figure 7 shows the time-conversion curves for the polymerization of DL-phenylalanine NCA initiated by $n$-hexylamine in the presence of various pyridine derivatives. $n$-Hexylamine was added to the mixture of DL-phenylalanine NCA and pyridines. The slow polymerization (up to $3-4-\%$ conversion) before the addition of $n$ hexylamine is due to the initiation by pyridine derivatives. A rapid polymerization ensues im- mediately upon the addition of $n$-hexylamine. In practice only the normal-amine-type polymerization is involved (see, for example, Table III), so the comparison of initial rates corresponds to the comparison of propagation rate constants.

The effect of pyridine derivatives on the nhexylamine-initiated polymerization was determined from Figure 7 and is shown in Table II. Additions of pyridine and the low concentration of $2,2^{\prime}$-bipyridine showed little influence, which is as expected. In the presence of the high concentration of $2,2^{\prime}$-bipyridine (matrix), the contribution of Scheme 3 is important and the acceleration was in fact observed. The retarding effect of 4,4'-bipyridine may be a result of nonproductive adsorption of NCA as illustrated in Scheme 4.

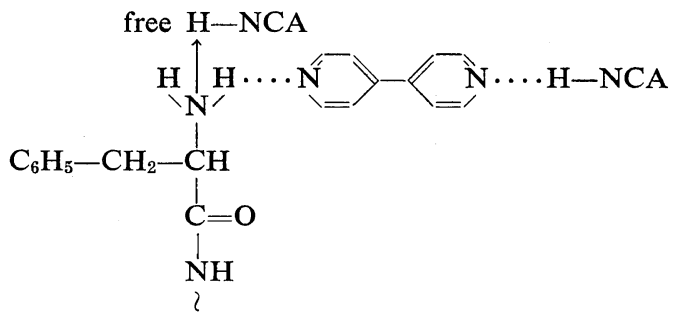

Scheme 4.

Poly(2-vinylpyridine) is one of the pyridine derivatives, but is unique in that pyridine groups are not conjugated with each other. The cooperation of the type shown in Scheme 1 is not expected for poly(2-vinylpyridine), but still an acceleration was observed. Possibly two or more of the many pyridine groups can assume a spatial arrangement suitable for the reaction.

\section{Molecular Weight of Polymer}

It is important to confirm that the polymeri-

Table II. Polymerization ${ }^{a}$ of DL-phenylalanine NCA by $n$-hexylamine in the presence of multifunctional pyridine derivatives

\begin{tabular}{lcccc}
\hline Pyridine derivatives & $\begin{array}{c}\text { Concentration, } \\
\text { mol } / l\end{array}$ & $\begin{array}{c}\text { Hydrogen-bonded } \\
\text { NCA, } \%\end{array}$ & $R_{\mathrm{p}} \times 10,{ }^{3} \mathrm{~mol} / l \mathrm{~min}$ & $R_{\mathrm{p}}$, relative \\
\hline- & - & - & 10.0 & 1 \\
Pyridine & 1.0 & 93.5 & 9.0 & 0.90 \\
2,2'-Bipyridine & 1.0 & 48.5 & 16.6 & 1.66 \\
$\quad$ " & 0.02 & 74.5 & 8.9 & 0.89 \\
4,4'-Bipyridine & 1.0 & 79.5 & 6.4 & 0.64 \\
Poly(2-vinylpyridine) & 1.0 & 13.4 & 1.34 \\
\hline
\end{tabular}

a In nitrobenzene at $35^{\circ} \mathrm{C},[\mathrm{NCA}], 0.2 \mathrm{~mol} / l$, [ $n$-hexylamine $]_{0}, 0.02 \mathrm{~mol} / l$. 
Table III. Molecular weight of poly(DL-phenylalanine) $)^{2}$

\begin{tabular}{lcc}
\hline \multicolumn{1}{c}{ Initiator } & $\begin{array}{c}\text { Concentration, } \\
\mathrm{mol} / l\end{array}$ & {$[\eta],{ }^{\mathrm{b}} \quad 100 \mathrm{ml} / \mathrm{g}$} \\
\hline Pyridine & 1.0 & 0.50 \\
" & 0.5 & 0.46 \\
" & 0.2 & 0.55 \\
2,2'-Bipyridine & 1.0 & 0.91 \\
2,2'-Bipyridine & 1.0 & \} 0.058 \\
$n$-Hexylamine & 0.02 &
\end{tabular}

a Polymerization in nitrobenzene at $35^{\circ} \mathrm{C},[\mathrm{NCA}]_{0}$, $0.2 \mathrm{~mol} / l$.

b Determined in $\mathrm{CHCl}_{3}-\mathrm{CHCl}_{2} \mathrm{COOH}(95: 5 \mathrm{v} / \mathrm{v})$ mixed solvent at $25^{\circ} \mathrm{C}$.

zations initiated by various pyridine derivatives are of the same mechanism (activated-NCA type), and are different from those initiated by $n$-hexylamine (normal-amine type). This is substantiated by the comparison of the solution viscosities of polymers formed (Table III). The viscosities of the polymers formed by pyridine and $2,2^{\prime}$-bipyridine are very much higher (typical for activated-NCA mechanism) than the viscosity of the polymer formed by $n$-hexylamine (typical for normal-amine-type mechanism). ${ }^{18}$

\section{REFERENCES}

1. K. Suzuoki, Y. Imanishi, T. Higashimura, and S. Okamura, Biopolymers, 7, 917 (1969).

2. K. Suzuoki, Y. Imanishi, T. Higashimura, and S. Okamura, ibid., 7, 925 (1969).
3. Y. Imanishi, S. Nagaoka, K. Suzuoki, and T. Higashimura, ibid., 12, 181 (1973).

4. M. Sisido, Y. Imanishi, and S. Okamura, ibid., 7, 937 (1969).

5. A. Patchornik and Y. Shalitin, Anal. Chem., 33, 1887 (1961).

6. C. H. Bamford and R. C. Price, Trans. Faraday Soc., 61, 2208 (1965).

7. C. H. Bamford and H. Block in "Polyamino Acids, Polypeptides, and Proteins," M. A. Stahmann, Ed., Wisconsin Univ. Press, Madison, Wisconsin, 1962, p 65.

8. M. Szwarc, Adv. Polymer Sci., 4, 1 (1965).

9. C. H. Bamford and H. Block, J. Chem. Soc., 4989 (1961).

10. H. C. Brown and X. R. Mihm, J. Amer. Chem. Soc., 77, 1723 (1955).

11. P. Krumholz, ibid., 73, 3487 (1951).

12. W. W. Brandt and J. P. Wright, ibid., 76, 3082 (1954).

13. "Dai Yuki Kagaku," Extra Vol. 2, M. Kotake, Ed., The Asakura Publishing Co., Ltd., Tokyo, 1963, p 598.

14. T. Kitajima, Thesis, Kyoto University, Kyoto, 1970.

15. S. Castellano, H. Günther, and S. Ebersole, $J$. Phys. Chem., 69, 4166 (1965).

16. T. Yonezawa, T. Nagata, H. Kato, A. Imamura, and K. Morokuma, "Ryoshi Kagaku Nyumon," Kagaku Dozin, Kyoto, 1972, p 56.

17. R. D. Brown and M. L. Hefferman, Aust. J. Chem., 9, 83 (1956); R. D. Brown and R. D. Harcourt, J. Chem. Soc., 3451 (1959).

18. D. G. H. Ballard and C. H. Bamford, J. Chem. Soc., 381 (1956). 